\title{
RITS acts in cis to promote RNA interference-mediated transcriptional and post-transcriptional silencing
}

\author{
Ken-ichi Noma ${ }^{1}$, Tomoyasu Sugiyama ${ }^{1}$, Hugh Cam $^{1}$, Andre Verdel ${ }^{2}$, Martin Zofall ${ }^{1}$, Songtao Jia ${ }^{1}$, \\ Danesh Moazed $^{2}$ \& Shiv I S Grewal ${ }^{1}$
}

RNA interference is a conserved mechanism by which double-stranded RNA is processed into short interfering RNAs (siRNAs) that can trigger both post-transcriptional and transcriptional gene silencing. In fission yeast, the RNA-induced initiation of transcriptional gene silencing (RITS) complex contains Dicer-generated siRNAs and is required for heterochromatic silencing. Here we show that RITS components, including Argonaute protein, bind to all known heterochromatic loci. At the mating-type region, RITS is recruited to the centromere-homologous repeat cenH in a Dicer-dependent manner, whereas the spreading of RITS across the entire 20-kb silenced domain, as well as its subsequent maintenance, requires heterochromatin machinery including Swi6 and occurs even in the absence of Dicer. Furthermore, our analyses suggest that RNA interference machinery operates in cis as a stable component of heterochromatic domains with RITS tethered to silenced loci by methylation of histone H3 at Lys9. This tethering promotes the processing of transcripts and generation of additional siRNAs for heterochromatin maintenance.

Heterochromatic chromosomal domains occur in a wide range of eukaryotes and have a crucial role in regulation of gene expression, sister chromatid cohesion and maintenance of genomic stability ${ }^{1,2}$. The genome of the fission yeast Schizosaccharomyces pombe contains large blocks of heterochromatin associated with pericentromeric repeats, telomeres and the silent mating-type region $(m a t)^{2}$. Heterochromatin assembly at these loci involves a conserved array of histone modifications that includes histone deacetylation and the Clr4mediated methylation of histone H3 at Lys9 (H3-Lys9), which is essential for the recruitment of chromodomain protein Swi6, a homolog of the fruit fly and mammalian HP1 proteins $s^{3-5}$.

The mechanisms that specify particular chromosomal regions as sites of heterochromatin assembly are not known. It is well known, however, that the main targets of heterochromatin assembly are DNA repetitive elements such as transposons and satellite repeats present at pericentric regions in large eukaryotic genomes ${ }^{2,6,7}$. The RNA interference (RNAi) pathway ${ }^{8,9}$ might be involved in heterochromatin nucleation at repeat loci ${ }^{10-15}$. In S. pombe, deletion of components of the RNAi pathway such as Argonaute (Ago1), Dicer (Dcr1) or RNA-dependent RNA polymerase (Rdp1) disrupts heterochromatinmediated silencing that correlates with loss of H3-Lys9 methylation and Swi6 association with heterochromatic $\operatorname{loci}^{14,15}$. In addition, siRNAs corresponding to centromeric repeats have been identified ${ }^{16}$, and the centromere-homologous cenH sequence ( $96 \%$ similar to $d g$ and $d h$ centromeric repeats ${ }^{17}$ ) found at the mat locus is an RNAidependent heterochromatin nucleation center ${ }^{14}$. An RNAi effector complex (RITS) containing Chp1, Tas3 and Ago1 is involved in heterochromatin assembly ${ }^{18}$. RITS contains siRNAs that are believed to serve as specificity determinants for targeting RNAi effector complexes to homologous sequences ${ }^{8,18-21}$.

In this study, we further dissected the role of RITS in heterochromatin assembly and silencing in S. pombe. We found that the three known RITS components, Agol, Chp1 and Tas3, bind to all known heterochromatic loci, including the mat locus, telomeres and centromeres. We also found that at the mat locus, RITS spreads from the heterochromatin nucleation site $c e n H$ to adjacent sequences, encompassing the entire heterochromatic mat region. We showed that RITS depends on H3-Lys9 methylation to remain stably associated with the silenced loci, and that the tethering of RITS to silenced chromosomal domains is essential for its role in transcriptional and post-transcriptional silencing and for the generation of additional siRNAs for heterochromatin assembly.

\section{RESULTS}

Localization of RITS components to heterochromatic loci

At least two components of RITS, Chp1 and Tas3, are associated with the centromeric repeats ${ }^{18,22}$. We carried out chromatin immunoprecipitation (ChIP) analysis to determine whether Agol also localizes to the centromeres and whether RITS components associate with the other heterochromatic loci, including the mat locus and telomeres. We found not only that Agol was readily detected at centromeric repeats, but also that all three known subunits of RITS complex were preferentially enriched at $c e n H$, the site of heterochromatin nucleation at the mat locus, and at the telomeres (Fig. 1a). Based on these

\footnotetext{
${ }^{1}$ Laboratory of Molecular Cell Biology, National Cancer Institute, National Institutes of Health, Bethesda, Maryland 20892, USA. ${ }^{2}$ Department of Cell Biology, Harvard Medical School, Boston, Massachusetts 02115, USA. Correspondence should be addressed to S.I.S.G. (grewals@mail.nih.gov).
}

Published online 10 October 2004; doi:10.1038/ng1452 
a

Mating-type locus

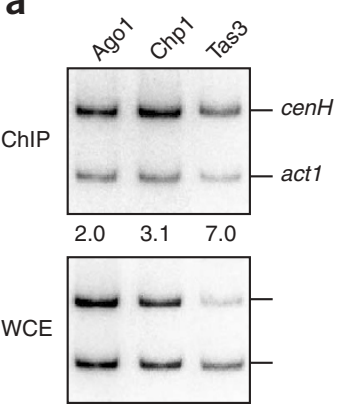

Centromere

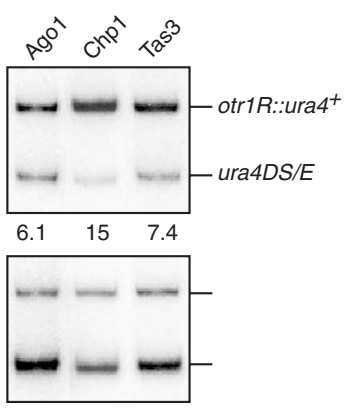

Telomere

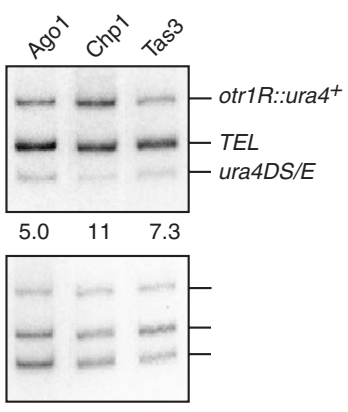

b

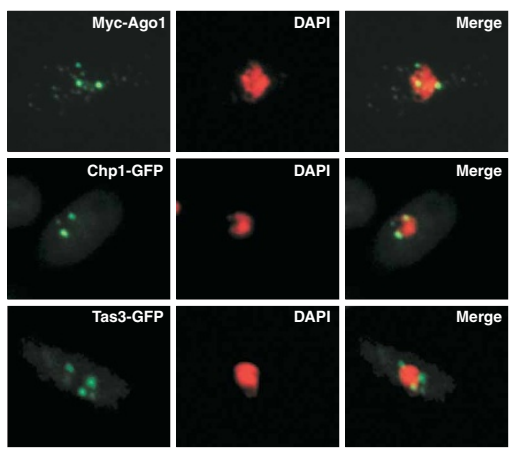

Figure 1 Stable association of RITS with constitutive heterochromatic domains. (a) RITS components are localized at the mating-type region, centromeres and telomeres. ChIP analyses were done using strains expressing $(\mathrm{Myc})_{3}-$ Ago 1, Chp1-(FLAG) $)_{3}$ and Tas3-TAP. Strains carrying ura4 ${ }^{+}$at centromeric repeat (otr1R:: ra $^{+}$) were used to detect RITS localization at centromeres. Telomeric region was amplified using primers previously described ${ }^{34}$. DNA isolated from ChIP or whole-cell extract (WCE) fractions was subjected to multiplex PCR to amplify DNA fragments from the mating-type region, centromeres or telomeres, along with an act1 or ura4DS/E fragment as an internal amplification control. The ratios of heterochromatic loci signals versus control signals between ChIP and whole-cell extract fractions were used to calculate the relative enrichment of precipitated samples ${ }^{35}$, shown below each lane. (b) Localization of RITS components as visualized by immunofluorescence with antibody to Myc (for (Myc) 3 -Ago1) or fluorescence of GFP-tagged proteins (for Chp1 and Tas3). DNA was visualized by DAPI.

analyses, RITS associates with known sites of heterochromatin formation in the fission yeast genome. Further supporting this idea, RITS subunits, including Agol, localized preferentially at two or three foci representing heterochromatic loci, including centromeres and telomeres, clustered near the nuclear periphery (Fig. 1b). We also observed speckled distribution of Agol in the cytoplasm, in addition to the bright foci present at the nuclear periphery. The meaning of the cytoplasmic localization of Ago1 remains to be investigated, but Agol might exist in multiple protein complexes with distinct functions.

\section{RITS-mediated heterochromatin assembly at the mat locus}

Although RITS is essential for heterochromatin assembly at centromeres, it is dispensable for the maintenance of the preassembled (2) heterochromatic state at the mat $\operatorname{locus}^{18}$. But RITS might be required for the initial nucleation of heterochromatin at the mat locus, as is the case with other factors involved in $\mathrm{RNAi}^{14}$. We previously showed that Atfl and Pcrl, two orthologs of mammalian ATF-CREB family proteins, act in a parallel mechanism to the RNAi pathway to nucleate heterochromatin assembly at the mat locus ${ }^{23}$, thereby compensating for the loss of RITS. To explore the possible role of RITS in heterochromatin assembly at the mat locus, we combined deletions of RITS components with pcr14. Deletion of $\operatorname{chp} 1$ or tas 3 alone had no effect on silencing of a $u r a 4^{+}$marker gene inserted at the mat locus (Kint2::ura4 $4^{+}$; Fig. 2a), but when combined with pcr14, deletion of either gene resulted in defective silencing of the $\mathrm{ura}^{+}$gene that correlated with a reduction in H3-Lys9 methylation and Swi6 localization (Fig. 2b). De novo nucleation of heterochromatin after treatment of cells with trichostatin A (TSA), a histone deacetylase inhibitor that 'erases' heterochromatin at the mat locus, was severely affected in double mutant cells a

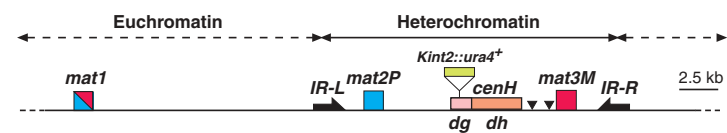

b

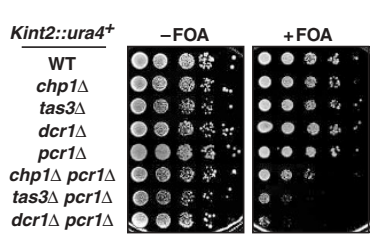

C
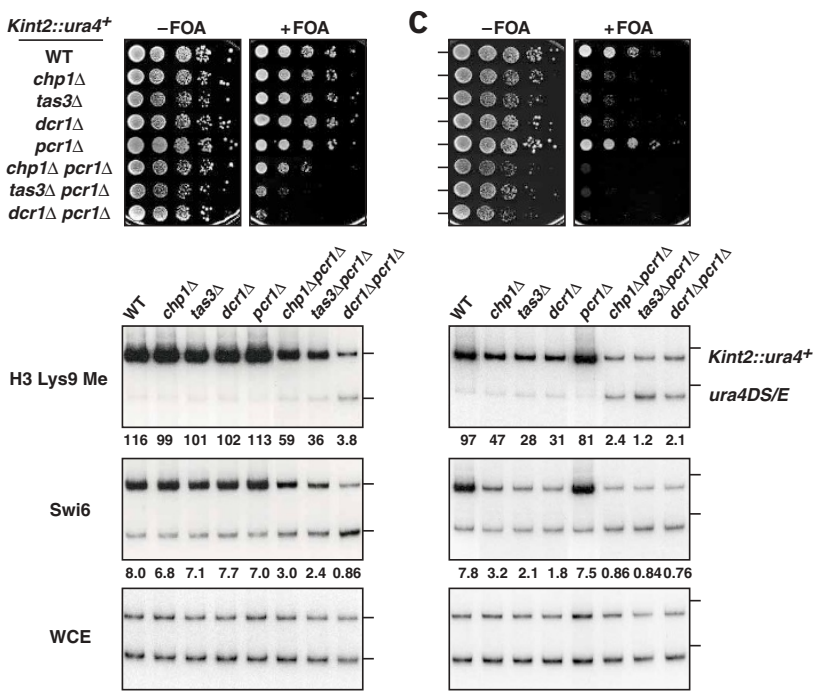

Figure 2 RITS cooperates with ATF-CREB family proteins to nucleate heterochromatin assembly at the mat locus. (a) Physical map of the mat region. mat1 is transcriptionally active, but a 20-kb domain encompassing mat 2 and mat 3 is subject to heterochromatin-mediated silencing. The inverted repeats $I R-L$ and $I R-R$ function as heterochromatin boundary elements. The cenH element shares homology to $d g$ and $d h$ centromeric repeats. Arrowheads indicate binding sites for Atf1-Pcr1. (b) Loss of RITS in combination with pcr1 $\Delta$ affects heterochromatin at Kint2::ura4 $4^{+}$. Serial dilution plating assays in the presence and absence of fluoroorotic acid (FOA) were done to measure Kint2::ura $4^{+}$ expression. Levels of H3-Lys9 methylation and Swi6 at Kint2::ura4 ${ }^{+}$were determined by ChIP assay. DNA from ChIP or whole-cell crude extracts (WCE) was analyzed using competitive PCR, whereby one primer pair amplifies different-sized PCR products from Kint2::ura4+ and from the control ura4DS/E minigene at the endogenous ura4 locus. The ratio of Kint2::ura4+ signal to ura4DS/E signal was used to calculate the relative enrichment, shown below each lane. (c) RITS mutant strains carrying pcr14 do not establish heterochromatin at the mat locus. Expression of Kint2::ura4+ and levels of H3-Lys9 methylation and Swi6 at Kint2::ura4+ after recovery from TSA treatment are shown. WT, wild-type. 

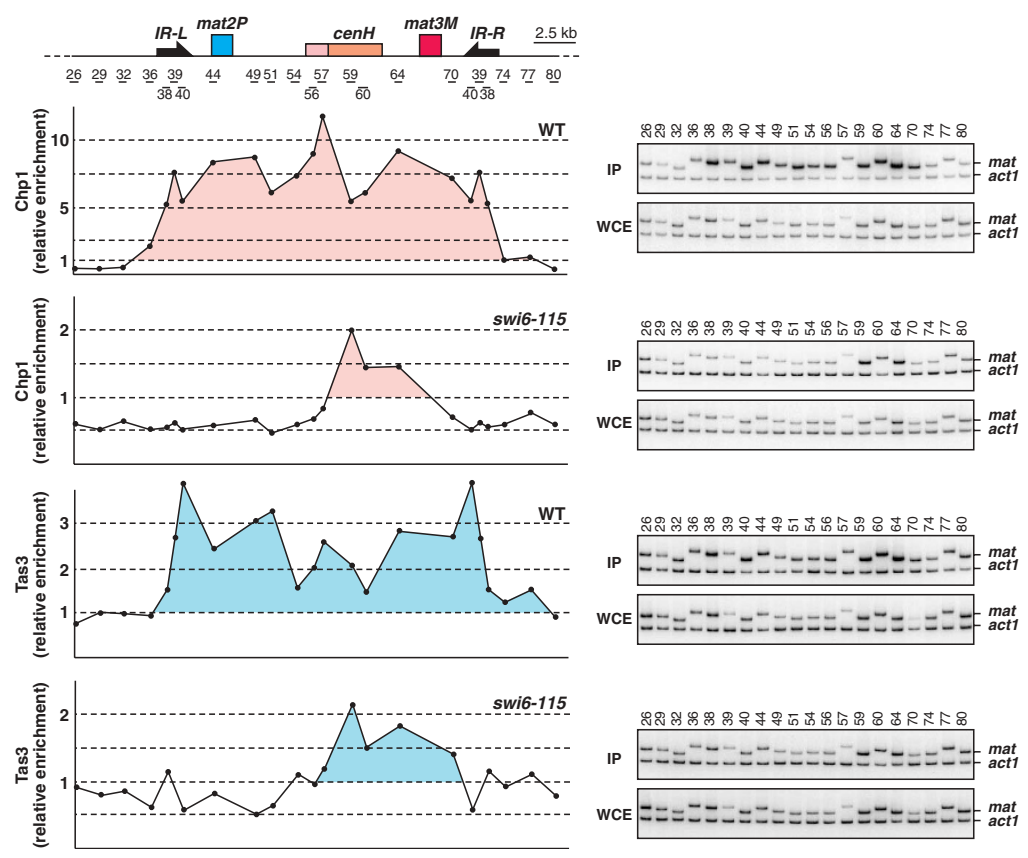

Figure 3 Mapping of RITS components at the mat region. ChIP was done using strains expressing Chp1-(hemagglutinin; $\mathrm{HA})_{3}$ or Tas3TAP with hemagglutinin-specific antibody (12CA5) or IgG sepharose, respectively. Primers used to amplify mat and control act 1 fragments were previously described ${ }^{35}$. DNA isolated from ChIP (IP) or whole-cell extract (WCE) fractions was subjected to multiplex PCR to amplify DNA fragments from the mat region (indicated by horizontal numbered bars), as well as an act 1 fragment as an internal amplification control. The ratios of the mat region and act 1 signals present in whole-cell extracts were used to calculate the relative enrichment of precipitated samples ${ }^{35}$. These results were plotted in alignment with a map of the mat locus. Enrichment by a factor of 1 is a baseline for no enrichment, with enrichment by a factor greater than 1 indicating protein binding (filled colors). ChIP experiments were repeated at least twice for each factor, and representative results are shown. WT, wild-type.
(Fig. 2c). Single mutants with deletions of $\operatorname{chp} 1$, tas 3 or $d c r 1$ could eventually assemble heterochromatin after treatment with TSA, but double mutants carrying deletion of $\operatorname{chp} 1$, tas 3 or $d c r 1$ together with deletion of pcrl were completely unable to nucleate heterochromatin (Fig. 2c). These results indicate that RITS and Atf1-Pcr1 participate in distinct and parallel pathways to nucleate heterochromatin at the mat locus.

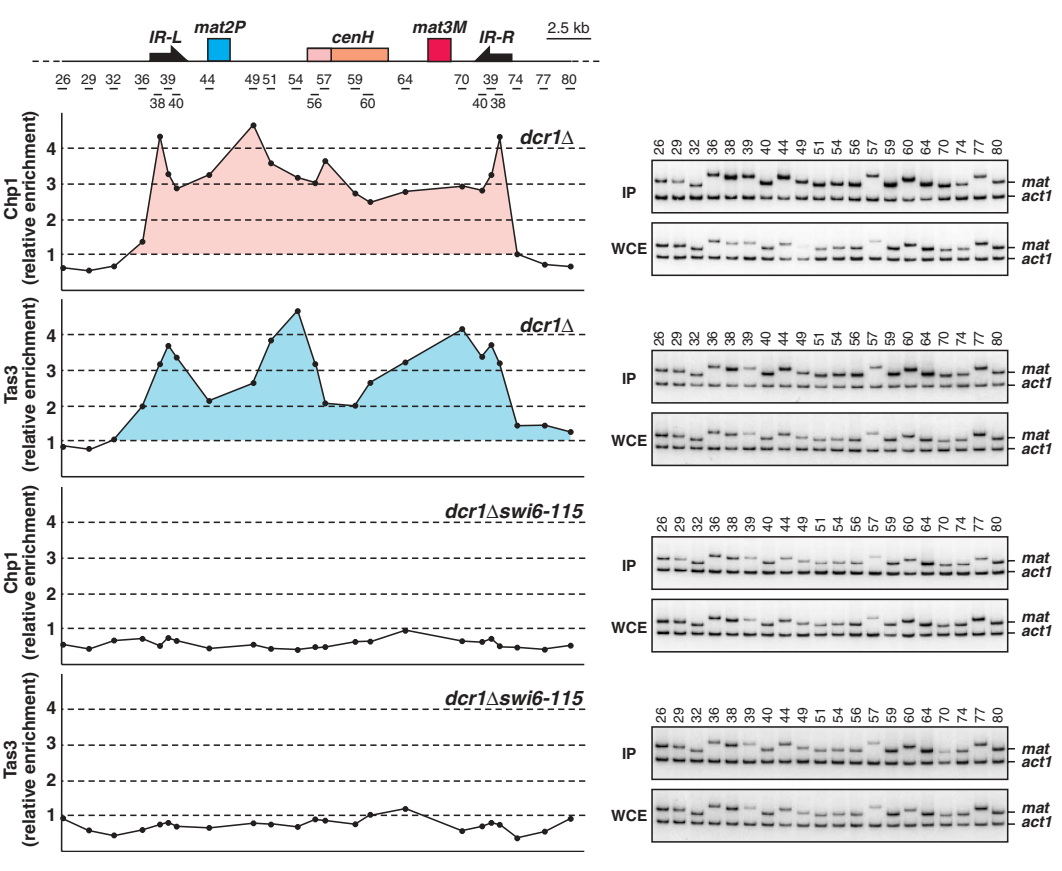

Figure 4 Localization of Chp1 and Tas3 at the mat region in dcr14 and dcr14 swi64 mutant cells. Levels of ChIP enrichment of Chp1-(FLAG) ${ }_{3}$ and Tas3-TAP proteins are presented in alignment with the map of the mat region. Quantitative measurements of Chp1-(FLAG) ${ }_{3}$ and Tas3-TAP protein levels at the mating-type region were carried out as described in Figure 3.

\section{Recruitment and spreading of RITS at the mat locus}

We next investigated whether RITS localizes specifically to cenH or is widely distributed throughout the mat region. High-resolution mapping of RITS components by ChIP assay showed that Chp1 and Tas3 were preferentially enriched throughout the entire $20-\mathrm{kb}$ mat region (Fig. 3). In swi6 mutant cells, which are able to nucleate heterochromatin but unable to spread H3-Lys9 methylation outward from the nucleation site ${ }^{14}$, RITS localization was restricted to the $c e n H$ heterochromatin nucleation center (Fig. 3). These results indicate that RITS localizes to cenH independent of Swi6 and suggest that heterochromatin containing Swi6 is required for the spreading and maintenance of RITS throughout the mat locus. RITS contains Dcrl-generated siRNAs that are believed to be essential for RITS localization to centromeric repeats ${ }^{18}$. We explored whether Dcr1 was also required for RITS localization to the mat locus. We found that RITS localization was not affected by the loss of Dcr1 (Fig. 4), probably because heterochromatin assembled by Atf1-Pcr1 pathway was able to maintain RITS at the mat locus in the absence of an intact RNAi pathway. In double mutant cells carrying deletions of dcrl and swi6, which lack heterochromatin at the mat locus, RITS localization was completely abolished across the entire mat interval (Fig. 4). These data suggest that siRNAs generated by Dcrl are required for the de novo targeting of RITS to the cenH site, but, once localized, H3-Lys9 methylation and Swi6-containing heterochromatin are sufficient to maintain RITS across the mat region (Fig. 3). 
a

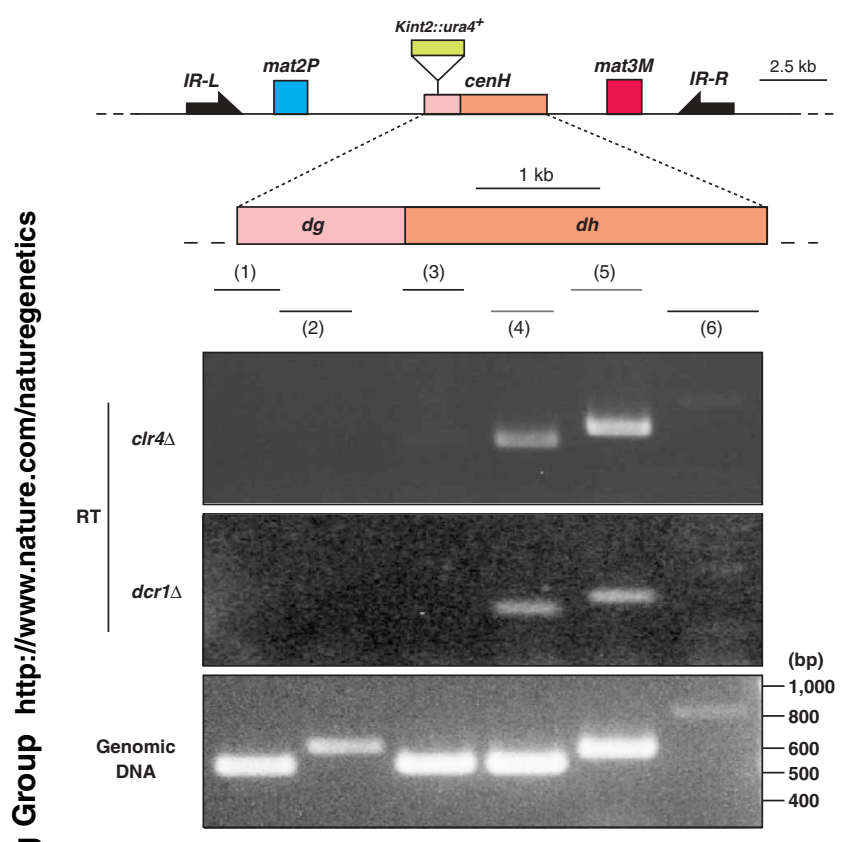

b
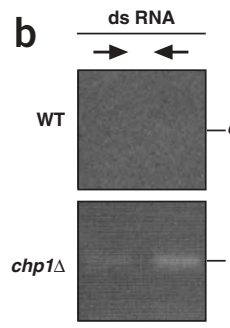

tas $3 \Delta$

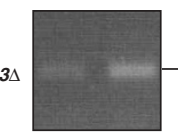

ago1 $\Delta$

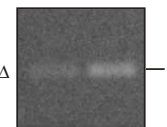

dcr1 $\triangle$

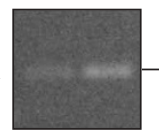

rdp1s

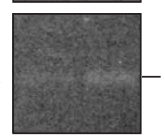

clr4 $\Delta$

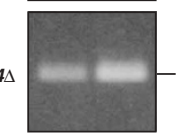

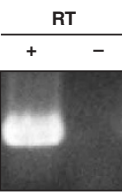
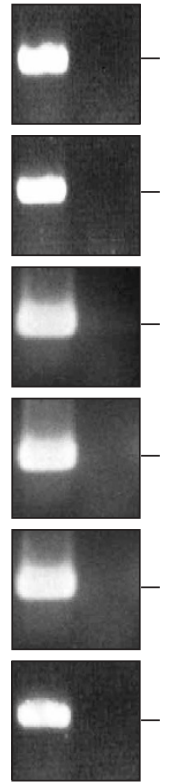

C

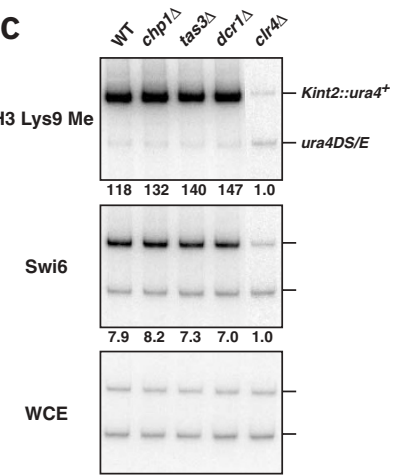

d

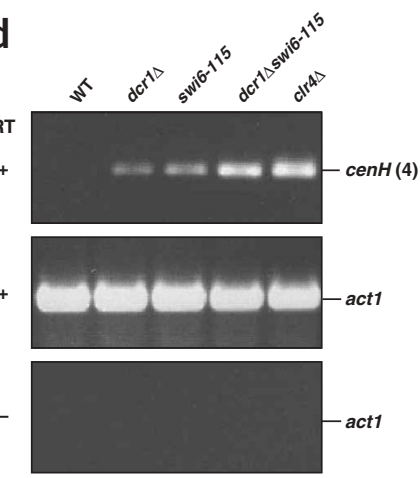

Figure 5 Bidirectional transcription of the cenH element and accumulation of the transcripts in RITS and other RNAi mutants. (a) Detection of transcripts from $c e n H$ in clr4A and $d c r 1 \Delta$ cells. RT-PCR was done using six primer pairs, which amplify different regions of cenH. PCR with genomic DNA template is shown as control (bottom). RT, reverse transcriptase. (b) Strand-specific RT-PCR was done as described previously ${ }^{15}$. Arrows indicate the direction of the primer used in first-strand cDNA synthesis. The same RNA samples used in $\mathbf{a}$ and $\mathbf{b}$ were also used to amplify control act1 transcript in the presence and absence of reverse transcriptase (RT). (c) Heterochromatin at Kint2::ura4+ is not affected by mutation in RITS or RNAi components. Levels of H3-Lys9

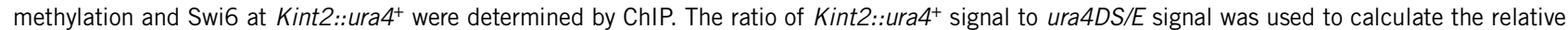
enrichment, shown below each lane. (d) The levels of cenH transcripts were analyzed in different genetic backgrounds by RT-PCR. WT, wild-type.

\section{RNAi and heterochromatin regulate cenH expression}

If Dcr1-generated siRNAs are required for heterochromatin nucleation at the mat locus, then cen $H$, like centromeric repeats, might be transcribed. We found that transcript levels were barely detectable in wild-type cells, whereas abundant transcripts corresponding to the $d h$ portion of $c e n H$ were readily identified in $d c r 1 \Delta$ and $c l r 4 \Delta$ strains (Fig. 5a). These transcripts were derived from both the forward and reverse strands of cenH (Fig. 5b), although the levels of forward transcripts were slightly higher, probably reflecting the relative strength of the respective promoters. Although deletions of RNAi pathway components had no effect on the maintenance of heterochromatin and silencing of marker genes inserted at the mat locus ${ }^{14}$ (Fig. 5c), they did result in a substantial accumulation of cenH transcripts. In this respect, $c e n H$ differs considerably from centromeric loci, where increased transcript levels in RNAi mutants are associated with a loss of heterochromatin and transcriptional silencing ${ }^{15}$. These findings suggest that double-stranded RNA (dsRNA) produced from $c e n H$ may be subject to post-transcriptional control in wild-type cells.

To elucidate the possible contributions of transcriptional and posttranscriptional mechanisms in the accumulation of cen $H$ transcripts, we examined the transcript levels in cells carrying deletions of $d \mathrm{cr} 1$, swi6, or both $d c r 1$ and swi6. Dcr1 is dispensable for heterochromatinmediated transcriptional silencing at the mat locus ${ }^{14}$ but is required for processing of dsRNA into siRNAs ${ }^{24}$. In contrast, Swi6 is essential for heterochromatin formation and transcriptional silencing but is not needed for targeting RITS to $c e n H$ (Fig. 3). Although dcr1 and swi6 single mutants each accumulated cen $H$ transcripts, $d c r 1$ swi6 double mutants accumulated substantially more transcripts (Fig. 5d). The levels of $c e n H$ transcripts in the double mutant were comparable to those in clr4A cells, which are defective in both heterochromatin assembly and RITS localization at cenH (Fig. 5d). These data suggest that the level of cen $H$ transcripts is regulated by both post-transcriptional and transcriptional silencing mechanisms.

\section{Targeting of RITS to heterochromatin and its role in silencing}

Localization of Chp1 to chromatin requires the Clr4 methyltransferase $^{22}$, which has also been implicated in the RNAi pathway ${ }^{21}$. We explored the interplay between Clr4-dependent localization of RITS to silenced loci and its effect on siRNA generation. To determine the role of Clr4 in RITS localization to heterochromatin, we carried out ChIP mapping of H3-Lys9 methylation and RITS at the mat locus in strains with mutations in either the chromodomain (W31G) or the post-SET domain (G486D) of Clr4 and in strains carrying the clr44 allele ${ }^{4}$. H3Lys9 methylation was present throughout the mat region in wild-type cells but was completely absent in the $c l r 4 \Delta$ cells (Fig. 6a) ${ }^{14}$. H3-Lys9 methylation was restricted to $c e n H$ in the G486D mutant and was asymmetrically enriched at cenH and sequences flanking to the right in the W31G mutant (Fig. 6a). Mapping of RITS at the mat locus showed a localization pattern markedly similar to the pattern of H3-Lys9 methylation in the $c l r 4$ mutants (Fig. 6b). RITS components localized to $c e n H$ region in the G486D and $\mathrm{W} 31 \mathrm{G}$ mutants, but neither Chp1 (Fig. 6b) nor Tas3 (data not shown) localized to the mat locus in clr44 
Figure 6 RITS localization correlates with H3-Lys9 methylation in clr4 mutants at the mat locus. H3-Lys9 methylation (a) and Chp1 localization (b) in clr4 mutants. Wild-type (WT), W31G, G486D and clr44 strains were used for ChIP analyses. W31G and G486D contain mutations in the chromodomain and post-SET domain of CIr4 protein, respectively. Levels of H3-Lys9 methylation and Chp1-TAP at the mat region determined by ChIP are shown aligned with the map of the mating-type region (Supplementary Fig. 2 online). cells. Similarly, deletion of clr4 abolished RITS binding to the centromeres (Supplementary Fig. 1 online). These data indicate that the localization of RITS at heterochromatic loci and its subsequent g spreading require H3-Lys9 methylation by Clr4.

We next examined the effects of mutations in clr4 on siRNAs that copurify with the RITS complex. Although protein components of the RITS complex remained associated together in all three clr4 mutant backgrounds (data not shown), deletion of clr4, which completely abolishes H3-Lys9 methylation and RITS binding to chromatin,

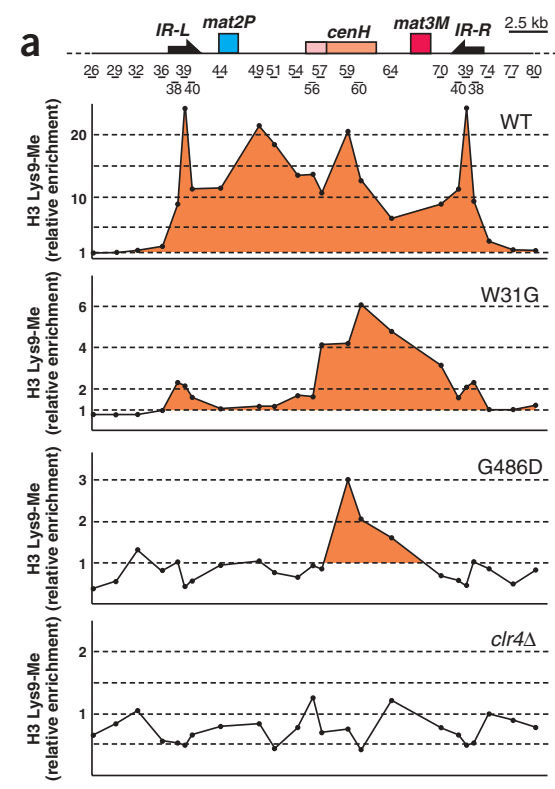

b
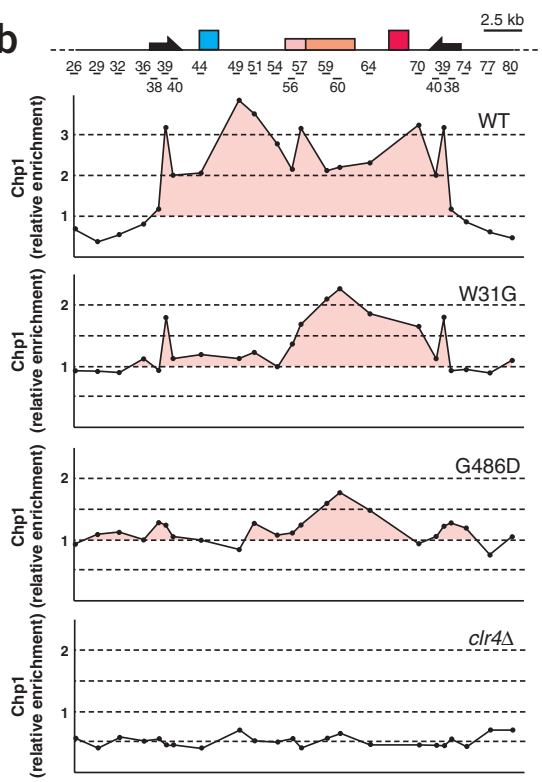

resulted in a loss of siRNAs associated with RITS (Fig. 7a). Consistent with RITS still being able to associate with heterochromatic repeats in the G486D and W31G mutants and with the presence of high levels of H3-Lys9 methylation at centromeres in these mutant backgrounds ${ }^{4}$, RITS-associated siRNAs were still present in both mutants (Fig. 7a). These results indicate that chromatin association of RITS is essential for efficient processing of transcripts at heterochromatic loci and for generation and recruitment of siRNAs into the RITS complex. To further explore this hypothesis, we constructed strains carrying a

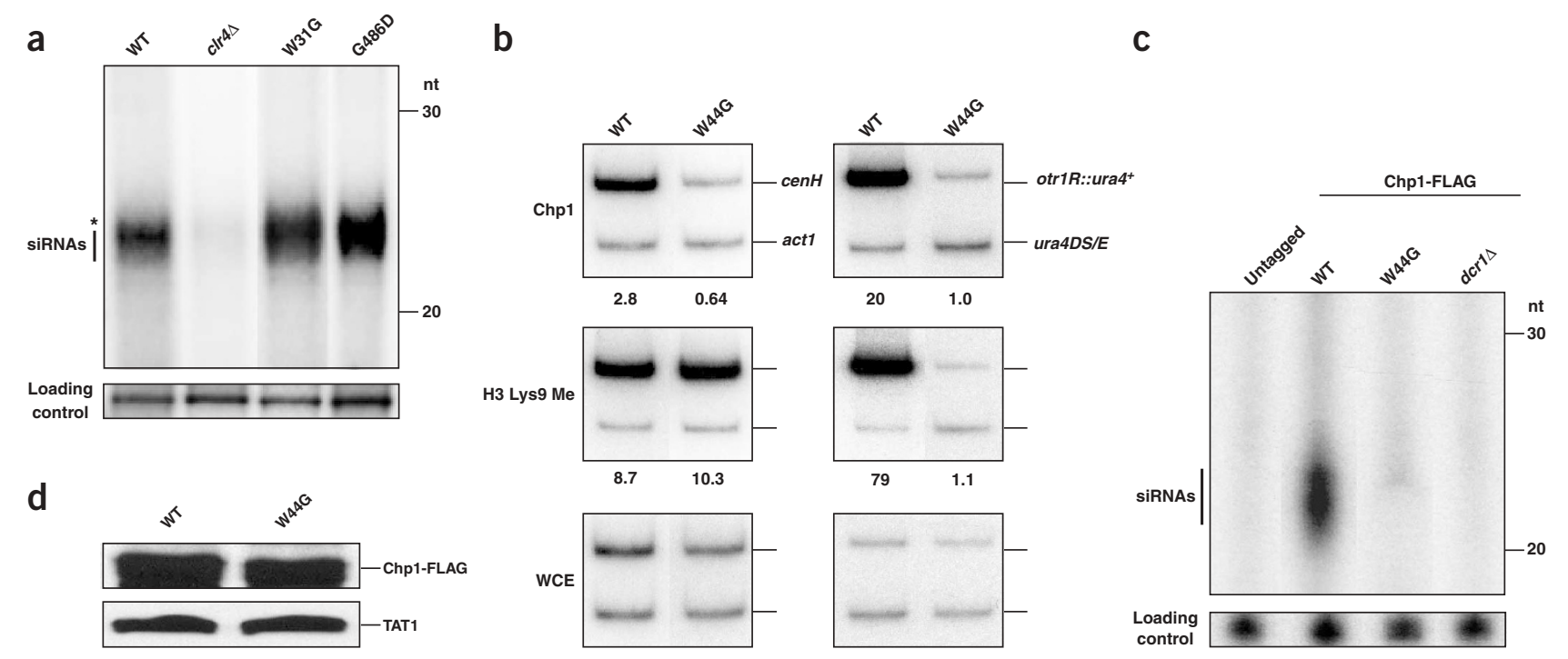

Figure 7 H3-Lys9 methylation-dependent stable association of RITS with silenced domains is essential for generation and recruitment of siRNAs into the complex and heterochromatin maintenance. (a) Effects of clr4 mutations on RITS-associated siRNAs. Chp1-TAP was purified from wild-type (WT) and clr4 mutant cells as described previously ${ }^{18}$. Asterisk indicates nonspecific background RNAs. A nonspecific RNA band present in all lanes is shown as a loading control. (b) The effect of mutation in Chp1 chromodomain on its localization and H3-Lys9 methylation at the mat region (left panels) and at the centromeres (right panels). Results from ChIP analyses assessing the effect of the W44G mutation on H3-Lys9 methylation and Chp1 localization are shown. (c) The effect of chp1 mutation on RITS-associated siRNAs. Strains carrying Chp1-(FLAG) $)_{3}$ were used in RITS purification. siRNAs in RITS were analyzed as described in a. (d) Comparison of Chp1 protein levels in wild-type (WT) and chp1 mutant cells, as assessed by western-blot analysis using antibody to FLAG (top panel) or to TAT1 for loading control (bottom panel). 


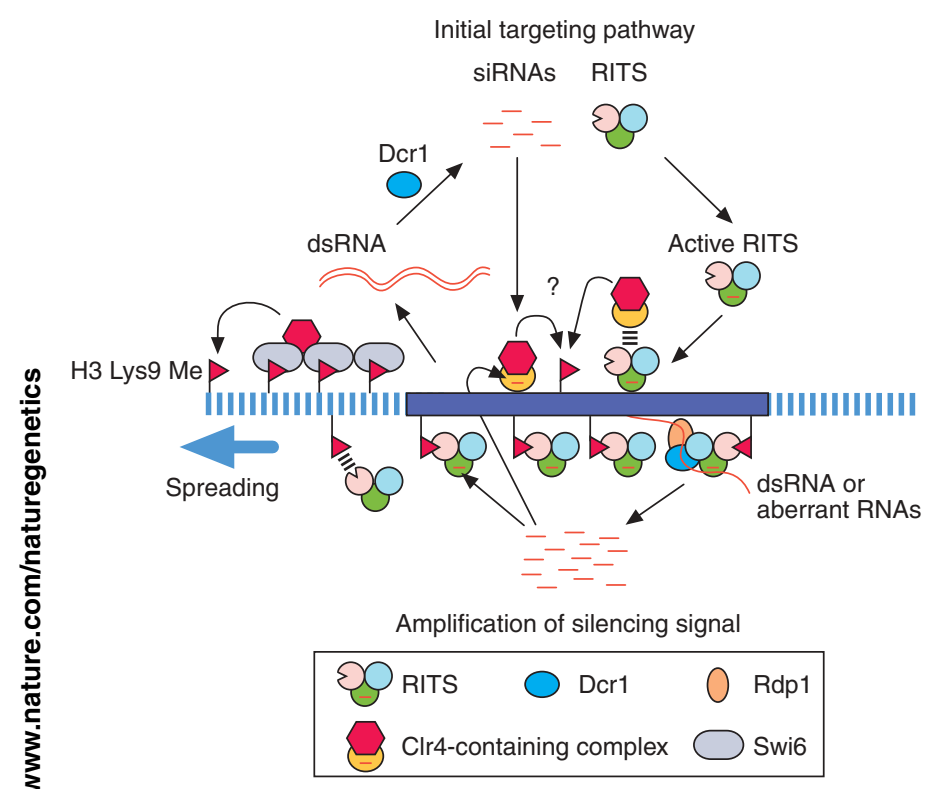

Figure 8 Schematic model showing the role of RITS and RNAi in transcriptional and post-transcriptional silencing. RNAi machinery is 을 involved in the initial targeting of $\mathrm{Clr} 4$ protein to heterochromatin nucleation center (blue box), producing dsRNAs through a potential interaction between ( Clr4 with RITS (bar symbol) or with an unknown complex containing siRNAs (top). After the initial methylation of H3-Lys9 by Clr4, RITS can be tethered to chromatin through the interaction of Chp1 chromodomain and methylated H3-Lys9. Once bound to chromatin, RITS probably recruits Rdp1 and Dcr1 to process nascent transcripts into siRNAs. Newly produced siRNAs can trigger further recruitment of $\mathrm{Clr} 4$, creating a feedback loop to stably maintain heterochromatin (bottom). Swi6 bound to methylated H3-Lys9 recruits histone-modifying activities, including $\mathrm{Clr} 4$, that create additional binding sites for $\mathrm{Chpl}$ chromodomain on adjacent nucleosomes, leading to RITS spreading.

mutation (W44G) in the Chp1 chromodomain, which has been shown to bind methylated H3-Lys9 (ref. 22). In the W44G mutant, RITS did not associate with the mat region and the centromeres (Fig. 7b) and, concomitantly, no RITS-associated siRNAs could be detected (Fig. 7c), even though mutant W44G Chp1 protein was expressed at normal levels (Fig. 7d). The lack of Chp1 binding to chromatin also resulted in loss of H3-Lys9 methylation at centromeres but not at the mat region (Fig. 7b), primarily due to redundant heterochromatin assembly mechanisms at the mat $\operatorname{locus}^{23}$ (Fig. 2). These results suggest that the failure of RITS to associate with chromatin leads not only to the disappearance of siRNAs associated with RITS but also to the loss of heterochromatin at centromeres, further supporting the idea that the RNAi machinery acts primarily in cis with RITS tethered to chromatin by methylated H3-Lys9.

\section{DISCUSSION}

Our analyses are suggestive of a self-enforcing loop mechanism by which heterochromatic marks originally established by the RNAi pathway maintain RITS at specific loci on chromatin. Our results also indicate that stably localized RITS mediates the processing of nascent RNA transcripts, thus degrading potentially deleterious transcripts and generating additional siRNAs for the further recruitment of $\mathrm{Clr} 4$ and heterochromatin assembly factors. As shown in our model (Fig. 8), diffusible dsRNA transcripts are initially recognized and cleaved by Dcrl, converting them into siRNAs that in turn are incorporated into RITS or an unknown complex that guides Clr4 and heterochromatin assembly factors to homologous target sequences, presumably through interactions with nascent RNA transcripts. A number of observations suggest that this initial targeting step, probably mediated by trans-acting RNAi factors, is rather inefficient: de novo centromere formation, which is linked to heterochromatin and depends on $d g$ and $d h$ repeats ${ }^{25}$ known to act through $\mathrm{RNAi}^{15}$, is a stochastic and inefficient process; and epigenetically 'active' centromeres, after transient inhibition of histone deacetylation by TSA treatment ${ }^{26}$ or propagation in an RNAi mutant background (I. Hall and S.I.S.G., unpublished data), can persist for many generations in otherwise wild-type cells. Once localized at the target site, however, methylation of H3-Lys9 by Clr4 creates the binding sites not only for Swi6 but also for RITS to be tethered to chromatin through the chromodomain of Chp1. The tethering of RITS to chromatin directly couples the generation of siRNAs to heterochromatin assembly and allows both transcriptional and post-transcriptional silencing to be maintained in a local, cis-acting manner. RITS may directly participate in the generation of siRNA signals by cooperating with Rdp1 and Dcr1 in cis to process nascent transcripts into siRNAs. This hypothesis is supported by recent evidence that the fruit fly Dicer proteins are components of the Argonaute-containing RNA-induced silencing complex (RISC) that is required for siRNA-directed mRNA cleavage $^{27,28}$, and in some organisms, such as in Arabidopsis, Dicer is localized to the nucleus ${ }^{29}$. Moreover, $\operatorname{Rdp} 1$ in $S$. pombe is preferentially enriched at the centromeric repeats ${ }^{15}$. RITS might be part of a larger RNAi complex containing all the components necessary for the conversion of transcripts into siRNAs. This holoenzyme complex could assemble on chromatin to coordinate with heterochromatin factors to effect post-transcriptional silencing.

We show here that RITS can spread from the initial nucleation site to surrounding sequences and that this process depends on Swi6 but does not require Dcr1. This probably occurs through the recruitment of Clr4 by chromatin-bound Swi6 to methylate H3-Lys9 residues on adjacent nucleosomes ${ }^{14,30}$, thereby allowing RITS to spread in a Dcrl-independent manner through the binding of the Chp1 chromodomain to methylated H3-Lys9. This stably localized RITS might provide a detection system for transcripts produced by repeat elements embedded in heterochromatic domains. We propose that H3-Lys9 methylation and heterochromatin are parts of an epigenetic marking system that preferentially targets RITS to transposons and repetitive elements. Notably, the existence of alternative, RNAi-independent recruitment mechanisms and the role of heterochromatic spreading in this process may allow RITS to exert control over DNA sequences incapable of triggering RNAi themselves. Considering the utility of coupling heterochromatin assembly with RITS localization, and considering that numerous siRNAs corresponding to repetitive elements and transposons have been identified in plants and animals ${ }^{31,32}$, similar mechanisms probably exist in other organisms as well.

\section{METHODS}

Fission yeast strains. We constructed $S$. pombe strains expressing Chp1 or Tas3 fused with epitope tags at the $\mathrm{C}$ terminus using a PCR-based module method ${ }^{33}$. We tagged Agol with a Myc epitope $(3 \times)$ at the $\mathrm{N}$ terminus. We expressed tagged chp1, tas 3 and agol under the control of their native regulatory elements so as to achieve wild-type levels of expression. The tagged proteins were fully functional as judged by centromeric silencing assay. We generated clr4 mutant strains with mutation in the chromodomain (W31G) or the post-SET domain (G486D) as described previously ${ }^{4}$. We created the chromodomain mutant of chpl by a PCR-based method using altered DNA sequence containing long 
oligonucleotides ( $\sim 100$ nucleotides) that corresponds to amino acid substitution W44G. We transformed the amplified PCR product into cells expressing Chp1-(FLAG) $)_{3}$. The presence of chp1 mutation was confirmed by DNA sequencing. All other strain constructions were carried out using conventional genetic crosses.

TSA treatment. We inoculated logarithmically growing cells in yeast extract supplemented with adenine (YEA) medium containing $35 \mu \mathrm{g} \mathrm{m}^{-1}$ TSA and cultured them for 10 generations at $32{ }^{\circ} \mathrm{C}$. We collected the cells, washed them twice with YEA medium and allowed them to grow for an additional 10 generations in the absence of TSA. We subjected TSA-treated and untreated cells to serial dilution plating assay as well as ChIP analyses.

ChIP. We carried out ChIP analysis with previously described primers ${ }^{34}$.

RNA analysis. We extracted total RNA from cells as described previously ${ }^{15}$, treated it $(\sim 10 \mu \mathrm{g})$ with 10 units of DNase I (Promega) at $37^{\circ} \mathrm{C}$ for $30 \mathrm{~min}$ and subjected the RNA sample ( $\sim 250 \mathrm{ng}$ ) to RT-PCR (Onestep RT-PCR kit, Qiagen). We detected dsRNA with one primer complementary to either the forward or reverse cenH transcripts during first-strand cDNA synthesis and added a second primer before the PCR reaction. To confirm that no DNA contamination was present in the RNA sample, we omitted the reverse transcription step.

Detection of RITS-associated siRNAs. We purified the RITS complex and detected RITS-associated siRNAs as previously described ${ }^{18}$. We grew cells carrying Chp1-TAP or Chp1-(FLAG) $)_{3}$ to mid-log phase in YEA medium and affinity-purified RITS from respective cell lysates. siRNAs purified by phenolchloroform extraction were then $3^{\prime}$-end-labeled with $\left[5^{\prime}-{ }^{32} \mathrm{P}\right]-\mathrm{pCp}$ using T4 RNA ligase. We resolved labeled siRNAs on a $15 \%$ denaturing urea acrylamide gel.

Immunofluorescence analysis. We carried out immunofluorescence analysis as previously described ${ }^{35}$

Note: Supplementary information is available on the Nature Genetics website.

\section{ACKNOWLEDGMENTS}

We thank I. Hall, J. Rice and B. Paterson for critical reading of the manuscript, 8 members of the laboratory of S.I.S.G. for discussions and Y. Tsukamoto for

technical assistance. Research in the laboratories of S.I.S.G. and D.M. is supported

(2) by the National Cancer Institute and the National Institutes of Health, respectively.

\section{COMPETING INTERESTS STATEMENT}

The authors declare that they have no competing financial interests.

Received 14 June; accepted 16 September 2004

Published online at http://www.nature.com/naturegenetics/

1. Grewal, S.I.S. \& Moazed, D. Heterochromatin and epigenetic control of gene expression. Science 301, 798-802 (2003).

2. Hall, I.M. \& Grewal, S.I.S. Structure and function of heterochromatin: implications for epigenetic gene silencing and genome organization. in RNAi: A Guide to Gene Silencing (ed. Hannon, G.) 205-232 (Cold Spring Harbor Laboratory Press, Cold Spring Harbor, 2003).

3. Richards, E.J. \& Elgin, S.C. Epigenetic codes for heterochromatin formation and silencing: rounding up the usual suspects. Cell 108, 489-500 (2002).

4. Nakayama, J., Rice, J.C., Strahl, B.D., Allis, C.D. \& Grewal, S.I.S. Role of histone H3 lysine 9 methylation in epigenetic control of heterochromatin assembly. Science $\mathbf{2 9 2}$ 110-113 (2001).
5. Bannister, A.J. et al. Selective recognition of methylated lysine 9 on histone $\mathrm{H} 3$ by the HP1 chromo domain. Nature 410, 120-124 (2001).

6. Hsieh, J. \& Fire, A. Recognition and silencing of repeated DNA. Ann. Rev. Genet. 34, 187-204 (2000).

7. Selker, E.U. et al. The methylated component of the Neurospora crassa genome. Nature 422, 893-897 (2003).

8. Hannon, G.J. RNA interference. Nature 418, 244-251 (2002).

9. Hutvagner, G. \& Zamore, P.D. RNAi: nature abhors a double-strand. Curr. Opin. Genet. Dev. 12, 225-232 (2002).

10. Zilberman, D., Cao, X. \& Jacobsen, S.E. ARGONAUTE4 control of locus-specific siRNA accumulation and DNA and histone methylation. Science 299, 716-719 (2003).

11. Mochizuki, K., Fine, N.A., Fujisawa, T. \& Gorovsky, M.A. Analysis of a piwi-related gene implicates small RNAs in genome rearrangement in Tetrahymena. Cell 110, 689-699 (2002).

12. Pal-Bhadra, M., Bhadra, U. \& Birchler, J.A. RNAi related mechanisms affect both transcriptional and posttranscriptional transgene silencing in Drosophila. Mol. Cel/ 9, 315-327 (2002).

13. Pal-Bhadra, M. et al. Heterochromatic silencing and HP1 localization in Drosophila are dependent on the RNAi machinery. Science 303, 669-672 (2004).

14. Hall, I.M. et al. Establishment and maintenance of a heterochromatin domain. Science 297, 2232-2237 (2002).

15. Volpe, T.A. et al. Regulation of heterochromatic silencing and histone $\mathrm{H} 3$ lysine-9 methylation by RNAi. Science 297, 1833-1837 (2002).

16. Reinhart, B.J. \& Bartel, D.P. Small RNAs correspond to centromere heterochromatic repeats. Science 297, 1831 (2002).

17. Grewal, S.I.S. \& Klar, A.J.S. A recombinationally repressed region between mat2 and mat3 loci shares homology to centromeric repeats and regulates directionality of mating-type switching in fission yeast. Genetics 146, 1221-1238 (1997).

18. Verdel, A. et al. RNAi-mediated targeting of heterochromatin by the RITS complex. Science 303, 672-676 (2004).

19. Hamilton, A.J. \& Baulcombe, D.C. A species of small antisense RNA in posttranscriptional gene silencing in plants. Science 286, 950-952 (1999).

20. Matzke, M., Matzke, A.J. \& Kooter, J.M. RNA: guiding gene silencing. Science 293, 1080-1083 (2001).

21. Schramke, V. \& Allshire, R. Hairpin RNAs and retrotransposon LTRs effect RNAi and chromatin-based gene silencing. Science 301, 1069-1074 (2003).

22. Partridge, J.F., Scott, K.S., Bannister, A.J., Kouzarides, T. \& Allshire, R.C. cis-acting DNA from fission yeast centromeres mediates histone $\mathrm{H} 3$ methylation and recruitment of silencing factors and cohesin to an ectopic site. Curr. Biol. 12, 1652-1660 (2002).

23. Jia, S., Noma, K. \& Grewal, S.I.S. RNAi-independent heterochromatin nucleation by the stress-activated ATF/CREB family proteins. Science 304, 1971-1976 (2004).

24. Provost, P. et al. Dicer is required for chromosome segregation and gene silencing in fission yeast cells. Proc. Natl. Acad. Sci. USA 99, 16648-16653 (2002).

25. Steiner, N.C. \& Clarke, L. A novel epigenetic effect can alter centromere function in fission yeast. Cell 79, 865-874 (1994).

26. Ekwall, K., Olsson, T., Turner, B.M., Cranston, G. \& Allshire, R.C. Transient inhibition of histone deacetylation alters the structural and functional imprint at fission yeast centromeres. Cel/ 91, 1021-1032 (1997).

27. Lee, Y.S. et al. Distinct roles for Drosophila Dicer-1 and Dicer-2 in the siRNA/miRNA silencing pathways. Cell 117, 69-81 (2004).

28. Pham, J.W., Pellino, J.L., Lee, Y.S., Carthew, R.W. \& Sontheimer, E.J. A Dicer-2dependent 80s complex cleaves targeted mRNAs during RNAi in Drosophila. Cel/ 117, 83-94 (2004).

29. Xie, Z. et al. Genetic and functional diversification of small RNA pathways in plants. PLoS Biol. 2, 642-652 (2004).

30. Schotta, G. et al. Central role of Drosophila SU(VAR)3-9 in histone H3-K9 methylation and heterochromatic gene silencing. EMBO J. 21, 1121-1131 (2002).

31. Llave, C., Kasschau, K.D., Rector, M.A. \& Carrington, J.C. Endogenous and silencingassociated small RNAs in plants. Plant Cell 14, 1605-1619 (2002).

32. Aravin, A.A. et al. The small RNA profile during Drosophila melanogaster development. Dev. Cell 5, 337-350 (2003).

33. Bahler, J. et al. Heterologous modules for efficient and versatile PCR-based gene targeting in Schizosaccharomyces pombe. Yeast 14, 943-951 (1998).

34. Nakayama, J., Allshire, R.C., Klar, A.J. \& Grewal, S.I.S. A role for DNA polymerase alpha in epigenetic control of transcriptional silencing in fission yeast. EMBO J. 20, 2857-2866 (2001).

35. Noma, K., Allis, C.D. \& Grewal, S.I.S. Transitions in distinct histone H3 methylation patterns at the heterochromatin domain boundaries. Science 293, 1150-1155 (2001). 